\title{
FORMATION OF INTERSTELLAR BUBBLE: A TIME DEPENDENT NUMERICAL SIMULATION
}

\author{
B. Aryal*, A. Devkota* and R. Weinberger** \\ *Central Department of Physics, Tribhuvan University, Kirtipur, Nepal. \\ **Institute of Astrophysics, Innsbruck University, Innsbruck, Austria.
}

\begin{abstract}
We present results of the numerical simulations for the first $10^{5}$ years of the development of spherically symmetric interstellar bubbles. We have assumed three phase interstellar medium (ISM) model and estimated the size of the interstellar bubbles (ISB). Our results are based on calculations including $10^{6}$ virtual stellar wind particles. We discuss the result in the context of Asymptotic Giant Branch (AGB) star, Proto-Planetary Nebula (PPN) phase and Relativistic Wind (RW) star. The size of the ISB is found 0.14 pc to 9.03 pc in the case of AGB (Asymptotic Giant Branch) star. This size increases for the PPN and RW phase to $0.64-40.28$ pc and 2.83 pc - 178.12 pc, respectively. It is found that the ISB can survive in the case of cold and warm interstellar medium. In the hot ISM, the ISB can not be formed due to the AGB wind. The bubble can survive in the case of PPN phase stellar wind for all kinds of the ISM. In addition, we found that the bubble can be formed in the case of RW when the ISM is hot. In the cold ISM, the ISB can not be formed due to the RW. In the warm ISM, bubble can be formed due to the relativistic (pulsar) wind if the mass loss rate is extremely high. In the warm ISM, the overall size of the bubble increases but do not exceed the recommended limit. Our result indicates that the hot ISM can not be considered as a good environment for the existence of the ISB. The size of the bubble exceeds the recommended limit (i.e., 1 to $5 \mathrm{kpc}$ ) in both the PPN and RW phase. However, suitable AGB wind can trigger the environment for a bubble in the hot ISM. The results of this work should be compared with the observations in the future.
\end{abstract}

Key words: Interstellar medium; Stellar wind; Planetary nebula; Pulsar.

\section{INTERACTION}

The space between the stars is referred to as the interstellar medium (ISM hereafter). By volume, the ISM is the largest "object” in the Milky Way Galaxy. Although the mass of the ISM is not known with certainty, the ISM has at least enough matter to make billions of stars like our Sun. The ISM is 99\% gas and $1 \%$ dust. Hydrogen is by far the most abundant element ( 90\%) in the ISM. The remaining $10 \%$ of the gas is composed of other elements, with helium having by far the greatest abundance. Carbon- and silicate-grains make up the dust component of the ISM, with particle sizes ranging from 0.1 - 1 micrometers, although some grains are considerably smaller. Over a hundred different molecules have been found to date in interstellar space, both in clouds and on interstellar grains.

Although the ISM has an average density of about 1 atom for every cubic centimeter, the distribution of atoms is far from uniform. Most conspicuous are the clouds of gas and dust that populate the ISM. Cosmic rays also traverse the ISM, and interstellar magnetic fields have been detected by radio astronomers ${ }^{1}$. Old stars die either in supernova explosions or by casting off material more gently and forming planetary nebulae. In either case the material released from a star's demise goes into the ISM. This material in turn, over millions of ears, finds its way into clouds of gas and dust from which other new stars subsequently form, stars which at the time of their own death will once again return to the ISM ${ }^{2}$.
Interstellar bubbles are the structures formed by the interaction between the fast stellar winds of star and ISM. These structures create an inner cavity surrounded by thin shell of swept up ionized gas which in turn may surrounded by neutral atoms or molecules. Weaver et al. ${ }^{3}$ proposed a model regarding the formation of interstellar bubble. However, they assumed homogeneous interstellar medium. It would be interesting if we make a model by considering inhomogeneous interstellar medium. We intend to consider three phase ISM (as proposed by Mackee \& Ostriker ${ }^{4}$ ) and improve the model proposed by Castor et al. ${ }^{5}$ regarding the formation of the interstellar bubble (ISB herafter). A numerical time dependent simulation is becoming a powerful tool in the field of interstellar interaction mechanism. We plan to perform a numerical time dependent simulations in order to estimate the sizes of the interstellar bubbles when stellar wind interacts with cold, warm and hot ISM.

\section{INTERSTELLAR BUBBLE}

When the fast winds of stars interact with ambient ISM, interstellar bubbles can be formed. These bubbles have an inner cavity surrounded by thin shell of swept up ionized gas. Discoveries of high velocity gas (up to 70 to $100 \mathrm{~km} \mathrm{~s}^{-1}$ ) in several diffuse nebulae, in particular around Wolf Rayet (WR) stars, for the first time formally established idea of presence of thin nebular shell formed of two layers: the shocked stellar wind and the shocked ambient medium separated by a contact discontinuity $^{6}$. A popular example of the ISB can be seen in Fig. 1.

Author for Correspondence: B. Aryal, Central Department of Physics, Tribhuvan University, Kirtipur, Nepal. 


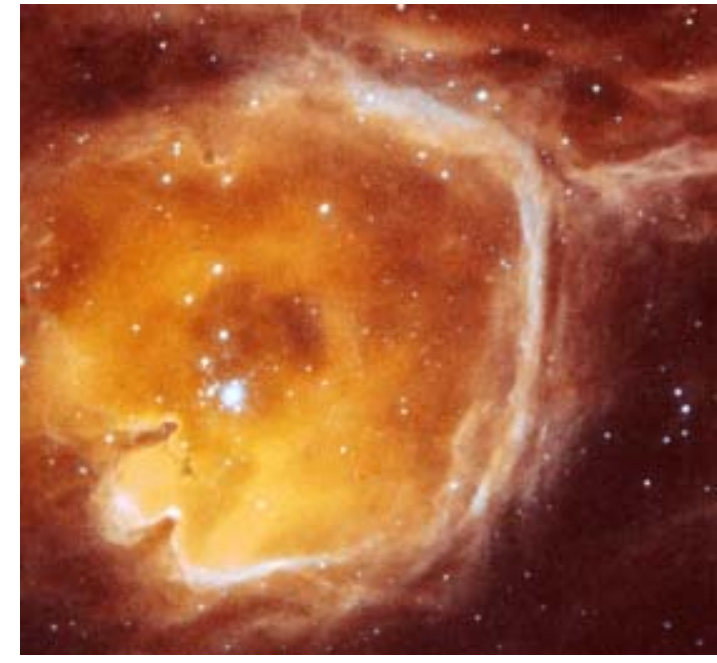

Figure 1: The Hubble Space Telescope captured this image of a 35light-year-wide interstellar bubble blown by radiation from a hot young star, known as N44F. (Y.H. Chu, University of Illinois, Urbana)

Dyson \& de Vries ${ }^{7}$ and Avedisova ${ }^{8}$ obtained similarity solution to this flow pattern and further developed by Falle? Weaver et al. ${ }^{3}$ made a comprehensive study of the flow pattern and gave a model which described the physical conditions and structure of bubble. The self similar solution gave the prediction which can be observed and hence it can be used to test the model. Most of the early models of bubbles assume that the stellar wind interacts with the ambient medium through momentum transfer and find that the shell expansion follows, where $r$ is the shell radius and $t$ is the dynamic age. Dyson \& de Vries ${ }^{7}$ were the first to suggest that the expansion of a bubble is driven by the thermal pressure of the shocked wind and follows the $\mathrm{r} \propto t^{3 / 5}$. Castor et al ${ }^{5}$, considering the thermal conduction through the discontinuity region and gave the basic structure of an interstellar bubble, which is divided into four regions (see Figure 2): (1) Region consists of freely expanding stellar wind moving with a velocity $(2000 \mathrm{~km} / \mathrm{s})$ enters shock which converts part of the energy into thermal energy (see region 'a' in Fig. 2). (2) Region containing shocked stellar wind, the sound speed in this region is very high (see region ' $b$ ' in Fig. 2). It is the expansion of this hot gas, drives the shell of shocked interstellar gas and looses energy by doing this work. Velocity very quickly drops to a value less than the internal sound speed. The sound crossing time becomes much less than the expansion timescale. The total energy of the region is determined by the energy lost in doing work against its surrounding and the energy fed through the internal shock. Because the expansion velocity is much less than the sounds speed, the energy in this region is almost entirely thermal. Since radiative cooling is extremely low in this region, this region is spatially extended. The high temperature $\left(10^{6} \mathrm{~K}\right)$ ensures that the elements are very highly ionized, which are not effective coolants and the cooling rate is appreciably lower.

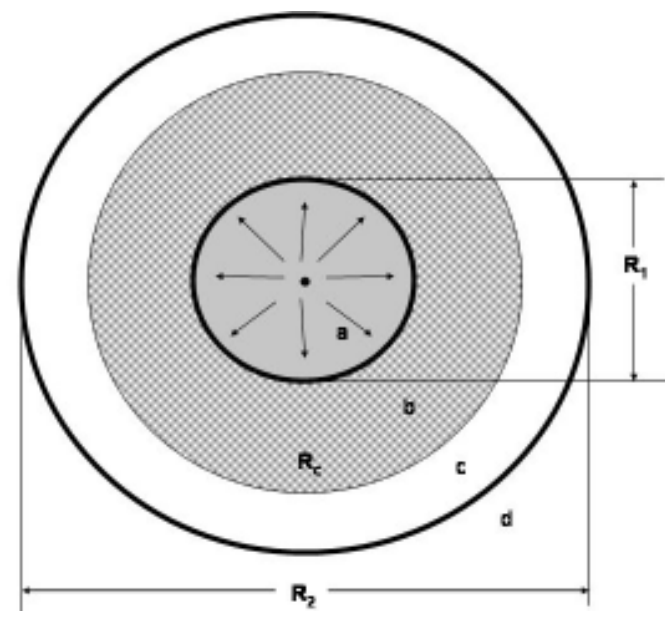

Figure 2: Interstellar bubble. The regions a, b, c and d represent the stellar wind, shocked stellar wind, shocked interstellar gas and ambient interstellar gas. The $R_{1}$ and $R_{2}$ represent the size of the ISB.

(3) This region consists of swept up interstellar medium (see region ' $c$ ' in Fig. 2). Radiative cooling is extremely effective in this region; therefore immediate post shock temperature is considerably lower than behind the inner shock. The gas in this region contains ions which can be collisionly excited by electrons to metastable states hence giving forbidden lines; therefore cooling is very effective in this region. This cooling increases the compression behind the outer shock; hence this region must be extremely thin. The gas cools down to a temperature $\left(10^{4} \mathrm{~K}\right)$ at which the cooling rate is equal to energy input rate due to photo ionization by the stellar radiation field. Therefore it has properties of a typical radiatively excited HII region (Aryal \& Weinberger ${ }^{10}$ ). Since the region is thin, and low sound velocity it is essentially at constant pressure. Across this surface the temperature, density and other physical characteristics of the gas change discontinuously. It is usually called a contact discontinuity. (4) The region of surrounding ambient interstellar medium (see region 'd' in Fig. 2).

Weaver et al. ${ }^{3}$ proposed a model regarding the existence of interstellar bubble due to the interaction between stellar wind and homogeneous interstellar medium. This wind interacts with an ambient interstellar gas of uniform atomic density $\rho_{\text {o }}$ and given cosmic abundances, resulting in an expanding spherical system, which we shall call a bubble. At first, the bubble is expanding so fast that radiative losses in the gas do not have time to affect any part of the system, and the dynamics of each region is described by adiabatic flow. In the second stage, radiative losses causes the expanding shell of swept-up interstellar gas in region (c) to collapse into a thin shell; but region (b), the shocked stellar wind, still conserves energy. In the final stage the radiative losses also affect the dynamics of region (b). Throughout the evolution of interstellar bubble, the dynamical system consists of four distinct zones. Staring from within, they are: (a) the hypersonic stellar wind; (b) a region of shocked interstellar wind; (c) a shell of shocked interstellar gas; and (d) ambient interstellar gas (see Fig. 2). Weaver et al. ${ }^{3}$ derived the expression for inner shocked region as follows: 
$R_{1}=0.90 \alpha^{3 / 2}\left(\frac{1}{\rho_{0}} \frac{d M_{w}}{d t}\right)^{3 / 10}\left(V_{w}\right)^{1 / 5} t^{2 / 5}$

$R_{2}=\left(\frac{0.406}{\pi}\right)^{1 / 5}\left(\frac{1}{\rho_{0}} \frac{d M_{w}}{d t}\right)^{1 / 5}\left(V_{w}\right)^{2 / 5} t^{3 / 5}$

Here $R_{1}$ and $R_{2}$ represent the inner shocked region and the boundary of the outer shocked region of the bubble, respectively. The $\alpha, V_{w}, t, d M_{w} / d t$ and $\rho_{o}$ represent the dynamical constant, wind velocity, evolution time, mass loss rate and atomic density of the medium, respectively. The dynamical constant corresponds to the proper motion of the structure. Figure 3 shows the size $\left(R_{1} \& R_{2}\right)$ versus mass loss rate and wind velocity plot. We can see changing slopes of $R_{1}$ and $R_{2}$.
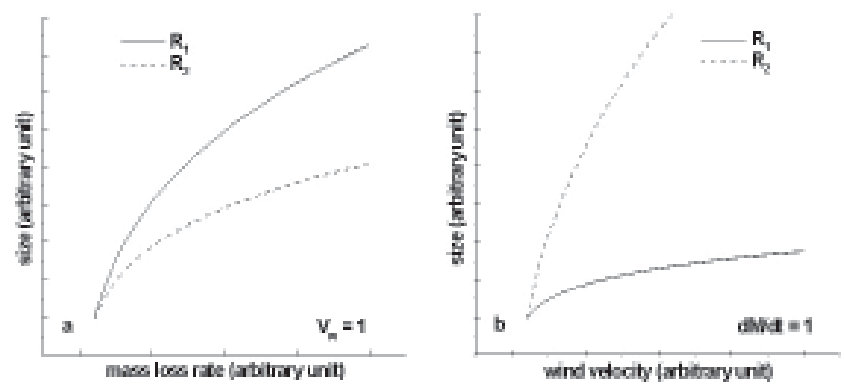

Figure 3: Size of the interstellar bubble $\left(R_{1}\right.$ and $\left.R_{2}\right)$ as a function of mass loss rate (a) and stellar wind velocity (b). The stellar wind velocity and the mass loss rate are assumed to be unity for (a) and (b), respectively. The evolution time of the interstellar bubble is assumed to be constant.

The model developed by Weaver et al. ${ }^{3}$ had the drawback that they assumed that the ISM surrounding the wind blowing star was homogeneous. One of the most important developments during 70's being the concept of 'multiphase' ISM. According to this concept, an ISM in which the gas can occupy any one of several states are in rough pressure equilibrium with the other states. In the years since it was proposed, three phase model (McKee \& Ostriker ${ }^{4}$ ) has been reasonably successful in accounting for a number of observed properties of the ISM and in providing several testable predictions. In their model, three phases interstellar gas exist. There is a hot, low-density gas ( $\mathrm{T} \sim 5 \times 10^{5} \mathrm{~K}$, density of $\mathrm{H}$ atom $\sim 0.004 \mathrm{~cm}^{-}$ ${ }^{3}$ ) that produces soft X-rays; a warm, moderate-density ( $\mathrm{T} \sim$ $10^{4} \mathrm{~K}$, density of $\mathrm{H}$ atom $\sim 0.3 \mathrm{~cm}^{-3}$ ), photo-ionized phase that accounts for the observed diffuse $\mathrm{H}_{\alpha}$ background; and a cold, high-density ( $\mathrm{T} \sim 100 \mathrm{~K}$, density of $\mathrm{H}$ atom $\sim 40 \mathrm{~cm}^{-3}$ ) phase that accounts for the observations of neutral gas. In this work, we intend to estimate the size of the interstellar bubble using random simulation. We use Weaver et al.'s (1977) prediction regarding the shocked stellar wind and shocked ISM and Mackee \& Ostriker's (1977) three-phase ISM model. The results of the simulation will be discussed in the context of Asymptotic Gaint Branch (AGB), Proto-Planetary Nebula (PPN and Relativistic Wind (RW) phase.

\section{SIMULATION PROCEDURE}

We run numerical time-dependent simulations for the first $10^{5}$ years of the development of spherically symmetric interstellar bubbles without considering electron thermal conduction. Our results are based on calculations including $10^{6}$ virtual stellar wind particles. Program file suitable for this purpose was written in MATLAB6.1. Our program file contains modified formulae for the radius of the inner shocked region $R_{1}$ (equation 1 ) and that of outer shocked region $R_{2}$ (equation 2). The program file and a sample of input file are given in appendix A and B. Our aim is to find the preferred size of the shocked stellar wind $\left(R_{1}\right)$ and the shocked interstellar medium $\left(R_{2}\right)$.

We have considered three phase interstellar medium (Mckee \& Ostriker 1977) that have following properties:

Cold Interstellar Medium: Density $=40 \mathrm{~cm}^{-3}$, Temperature $=$ $100 \mathrm{~K}$

Warm Interstellar Medium: Density $=0.3 \mathrm{~cm}^{-3}$, Temperature $=$ $10^{4} \mathrm{~K}$

Hot Interstellar Medium: Density $=0.004 \mathrm{~cm}^{-3}$, Temperature $=$ $5 \times 10^{5} \mathrm{~K}$

Here we present the result of the simulation. Figure 4 shows the distribution of inner shocked region $R_{1}$ and the outer shocked region $R_{2}$ against the number. This is the case when an ambient cold ISM interact with the wind that have velocity $<50 \mathrm{~km} \mathrm{~s}^{-1}$ (i.e., the AGB phase). This simulation is for the $10^{5}$ years time scale. A typical mass loss rate is chosen to be $10^{-4}$ to $10^{-5} \mathrm{M}_{\text {sun }}$ /year.
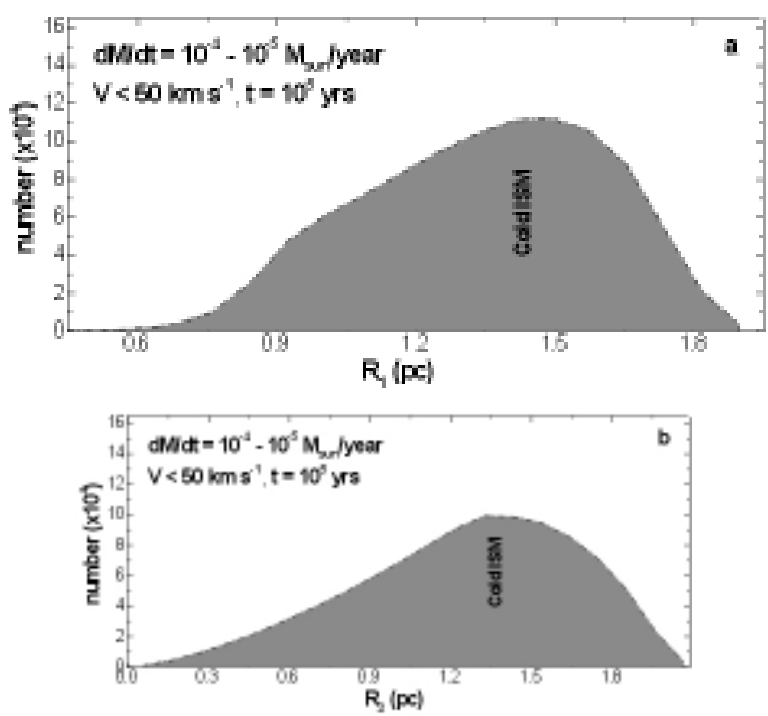

Figure 4: A comparison of inner shocked region $\left(R_{1}\right)$ and outer shocked region $\left(R_{2}\right)$ of the interstellar bubble $(\mathrm{a}, \mathrm{b})$ when stellar wind velocity is restricted to $50 \mathrm{~km} \mathrm{~s}^{-1}$, suitable for the ABG wind. Interstellar medium is supposed to be cold. The mass loss rate is given.

The grey-shaded region represents the region of interest. The full width half maximum (FWHM) is considered as the most preferred region. The FWHM is found to be wider for $R_{1}$ than that of $R_{2}$ (Fig. 4). This indicates that the shocked stellar wind is relatively prominent than that of the shocked interstellar medium. In the case of cold interstellar wind the preferred size $\left(R_{2}\right)$ of the bubble is found to be $\sim 0.89$ times than that of the inner shocked region $\left(R_{1}\right)$. 
Similarly curves for $R_{1}$ and $R_{2}$ for relativistic wind phase stars in hot ISM are shown in Fig. 5. The mass loss rate and the evolution time are the same as above. Here the most preferred size of the bubble is found about $\sim 178.12$ pc. However this is merely 5.56 times that of the size of the inner shocked region (i.e., $R_{1}$ ).

In this way, we have performed 54 simulations. The results of these simulations are discussed in the context of AGB, PPN and RW phase stars in the next chapter.
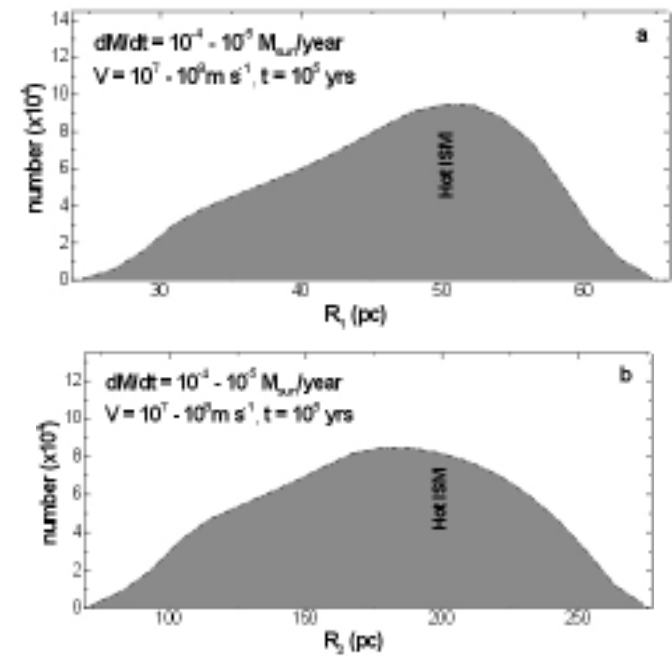

Figure 5: A comparison of inner shocked region $\left(R_{1}\right)$ and outer shocked region $\left(R_{2}\right)$ of the interstellar bubble when stellar wind velocity is restricted to $10^{7}-10^{8} \mathrm{~m} \mathrm{~s}^{-1}$, suitable for the relativistic wind. Interstellar medium is supposed to be hot. The mass loss rate is given.

\section{RESULTS}

Below we present the results of the random simulations regarding the existence of the cold, warm and the hot ISB in the AGB, PPN and RW phase.

\section{AGB Phase}

A period of stellar evolution undertaken by all low to intermediate mass stars (0.6-10 solar masses) late in their life is called Asymptotic Giant Branch (AGB) phase. When a star exhausts the supply of hydrogen in its core, the core contracts and its temperature increases, causing the outer layers of the star to expand and cool. Stars at this stage of stellar evolution are known as AGB stars. AGB stars are typically long period variables, and suffer large mass loss in the form of a stellar wind. A star may lose $50 \%$ to $70 \%$ of its mass during the AGB phase (Weinberger \& Kerber 1997). The stellar winds from AGB stars are the main production sites of dust in the universe. The AGB stars emit the particles as a stellar wind with a velocity of 5 - 75 $\mathrm{km} \mathrm{s}^{-1}$. Figure 6 shows the result of the numerical simulation regarding the size of the inner shocked region $\left(R_{1}\right)$ of the ISB formed due to AGB wind in the cold, warm and hot ISM.
A comparison between the sizes of inner shocked region $\left(R_{1}\right)$ in the cold, warm and hot ISM when the mass loss rate is $10^{-}$ ${ }^{4}$ to $10^{-5} \mathrm{M}_{\text {sun }}$ /year can be seen in Fig. 6a. The peaks of the distributions represent the preferred size for $R_{1}$. For cold ISM, the most preferable size of the inner shocked region, i.e., $R_{1}$, is found to be 1.49 pc. This value turns 6.47 pc and 23.78 pc for warm and hot ISM, respectively. As expected, the size of the inner shocked region is big when the AGB wind interacts with the hot ISM.

The peak value of $R_{1}$ is found $0.74 \mathrm{pc}, 3.24 \mathrm{pc}$ and $11.74 \mathrm{pc}$ for cold, warm and hot ISM when the mass loss rate decreased to $10^{-5}$ to $10^{-6} \mathrm{M}_{\text {sun }}$ /year (Fig. 6b). Similarly, for $10^{-6}$ to $10^{-7} \mathrm{M}_{\text {sun }}$ ' year, the size of the inner shocked regions are estimated as 0.37 pc, 1.61 pc and 5.88 pc for cold, warm and hot ISM, respectively (Fig. 6c). Thus, it is found that the size of the inner shocked region $\left(R_{1}\right)$ decreases with the decrease of mass loss rate. This tendency can be seen in the figures 6a-f. However, the nature of the distributions is found to be similar. The stability of ISB can be studied by estimating the full width half maxima (FWHM) of the spectrum. More stable ISB should have maximum value of FWHM. Thus, the hot ISB seem to be unstable. Interestingly, no overlapping region between three phases of the interstellar medium can be seen (Figs. 6a-f). This might indicate the fact that the evolution time, i.e., $\sim 10^{5}$ years, is reliable.

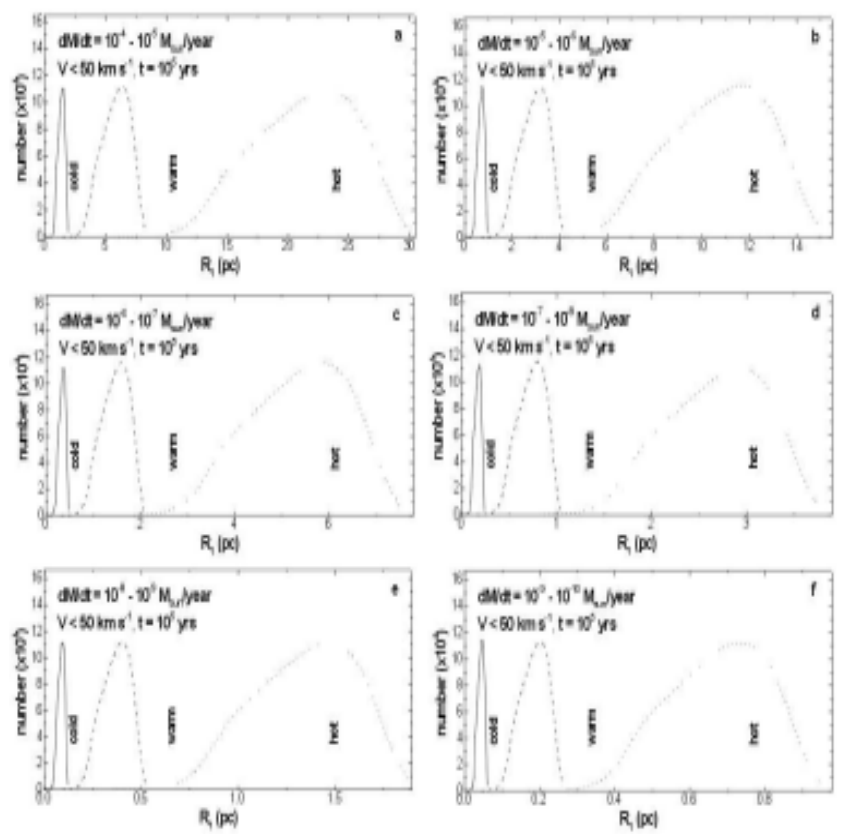

Figure 6: Size distribution of inner shocked region $\left(R_{1}\right)$ of the ISB formed due to AGB wind (50 km s$\left.{ }^{-1}\right)$ in cold (solid line), warm (dashed line) and hot (dotted line) ISM. The mass loss rates are given.

Now we discuss $R_{2}$ distribution. The $R_{2}$ represents the overall size of the bubble. Figure 7 represents $R_{2}$ distribution of the ISB in the cold, warm and hot ISM. 


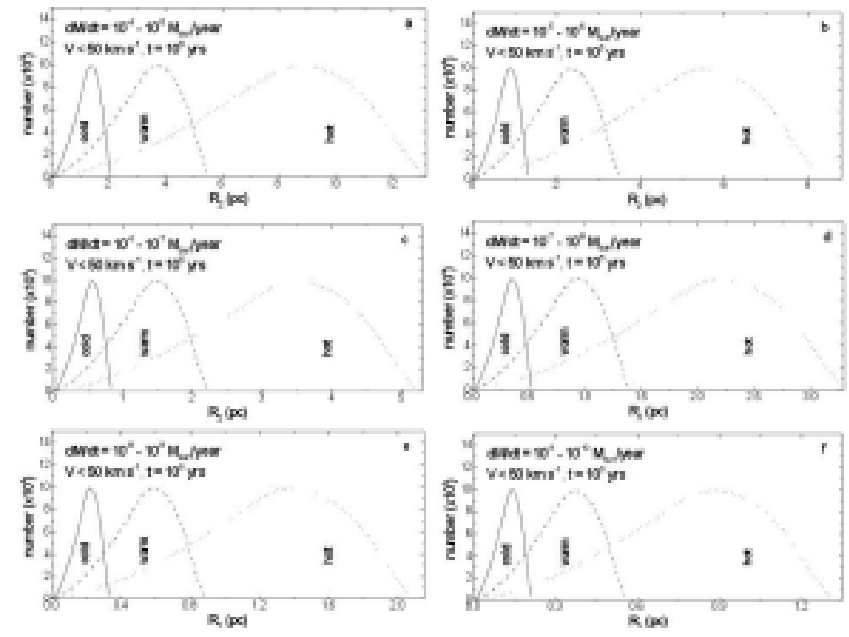

Figure 7: Size distribution of outer shocked region $\left(R_{2}\right)$ of the ISB formed due to AGB wind $\left(50 \mathrm{~km} \mathrm{~s}^{-1}\right)$ in cold (solid line), warm (dashed line) and hot (dotted line) ISM. The mass loss rates are given.

The peak value of the curve indicates the most probable value. As seen in Fig. 7 a the peak value for the mass rate $10^{-4}$ to $10^{-}$ ${ }^{5} \mathrm{M}_{\text {sun }}$ /year are 1.33 pc, $3.81 \mathrm{pc}$ and 9.03 pc for cold, warm and hot ISM, respectively. As expected, size of the bubble increases gradually from cold to hot ISM. The $R_{2}$ for the lower mass loss rates follow the similar trend as observed for mass loss rate $10^{-4}$ to $10^{-5} \mathrm{M}_{\text {sun }}$ /year in Fig. 7a. The peak value for the mass loss rate $10^{-5}$ to $10^{-6} \mathrm{M}_{\text {sun }}$ /year is found to be $0.84 \mathrm{pc}$ for cold region, $2.23 \mathrm{pc}$ for warm region and $5.28 \mathrm{pc}$ for hot region (Fig. 7). These values turned to be 0.57 pc, 1.41 pc and 3.59 pc for cold, warm and hot ISM for mass loss rate $10^{-6}$ to $10^{-7} \mathrm{M}_{\text {sun }}$ ' year (Fig. 7c). It is very much interesting to note the overlapping of curves for hot and warm medium with that of the cold medium. This suggests that hotter bubbles are less stable than colder bubbles.

Their ratio $\left(R_{2} / R_{1}\right)$ is found to be $>3$ in the case of cold ISM when the mass loss rate becomes $10^{-9}$ to $10^{-10} \mathrm{M}_{\text {sun }} /$ year. Interestingly, this ratio decreases with the increase of temperature. In most cases, this ratio is found to be $<1.0$ in hot ISM, indicating instability of the bubble. However, the formation of the ISB can not be denied. The preferred mass loss rate from the AGB wind lie in the range $10^{-7}$ to $10^{-10} \mathrm{M}_{\text {sun }}$ /year. In such cases, the ratio $R_{2} / R_{1}$ is found to be greater than 1.0 in cold and warm ISM. Thus, we conclude that the bubble can be survived in the case of AGB wind if the ISM is cold or warm. In hot ISM, the interstellar bubble can not be formed due to the AGB wind.

\section{PPN Phase}

The name protoplanetary nebula is a consequence of the older term planetary nebula, which was chosen due to early astronomers looking through telescopes and finding a similarity in appearance of planetary nebula to the gas giants such as Neptune and Uranus. To avoid any possible confusion, Sahai et al. (2005) suggests employing a new term pre-planetary nebula which does not overlap with any other disciplines of astronomy. They are often referred to as post-AGB star, although that category also includes stars that will never ionize their ejected matter. During this phase, the central star is still too cool to ionize the slow-moving circum-stellar shell ejected during the preceding AGB phase. However, the central star does appear to drive high-velocity, collimated winds which shape and shock this shell, and almost certainly entrain slow-moving AGB ejecta to produce a fast molecular wind. Protoplanetary Nebula (PPN) phase star emits stellar wind having velocity $1,000 \mathrm{~km} \mathrm{~s}^{-1}$. We used the method of numerical simulations to find sizes $\left(R_{1} \& R_{2}\right)$ of the ISB. Below we present the results of numerical simulations for cold, warm, and hot ISM (Fig. 8).

In PPN phase, the preferred value of the radius $R_{1}$ is found to be 2.30 pc for cold ISM when the mass loss rate $10^{-4}$ to $10^{-5}$ $\mathrm{M}_{\text {sun }}$ /year (Fig. 8a). The most preferred value (i.e., peak value) for warm ISM for the same mass loss rate turned out to be 9.96 pc and that for hot medium is 36.39 pc. The peak value of $R_{1}$ increases with the decrease in density of the ISM, as expected. The uniformity in the distribution curves is noticeable in the PPN phase. The $R_{1}$ is found to be 1.15 pc, 4.99 pc and 18.23 pc and for cold warm and hot ISM when the mass loss rate from the PPN phase star becomes $10^{-5}$ to $10^{-6} \mathrm{M}_{\text {sun }}$ /year (Fig. 8b). The most preferred value slightly decreases as the mass loss rate decreases. Similarly the inner shocked region $\left(R_{1}\right)$ are found $0.58 \mathrm{pc}, 2.50 \mathrm{pc}$ and $9.14 \mathrm{pc}$ for cold, warm and hot ISM, respectively when the mass loss rate is $10^{-6}$ to $10^{-7} \mathrm{M}_{\text {sun }}$ /year (Fig. 8c). Similar to the AGB phase, the distribution curves do not overlap with each other (Fig. 8). The distribution curves for cold ISM are narrower than that for warm ISM. The curves for hot ISM are wider than that for warm ISM. This suggests that the hot ISBs are less likely to form than the warm and cold bubbles.

The radius of the outer shocked region $R_{2}$ for different mediums is shown in Fig. 9. The radius of the bubble for cold, warm, and hot ISM for the mass loss rate $10^{-4}$ to $10^{-5} \mathrm{M}_{\text {sun }}$ /year are found to be 6.38 pc, 16.98 pc, and 40.28 pc, respectively (Fig. $9 a)$. Increase in radius of the bubble from cold to hot medium is due to decrease in density (or increase in temperature).
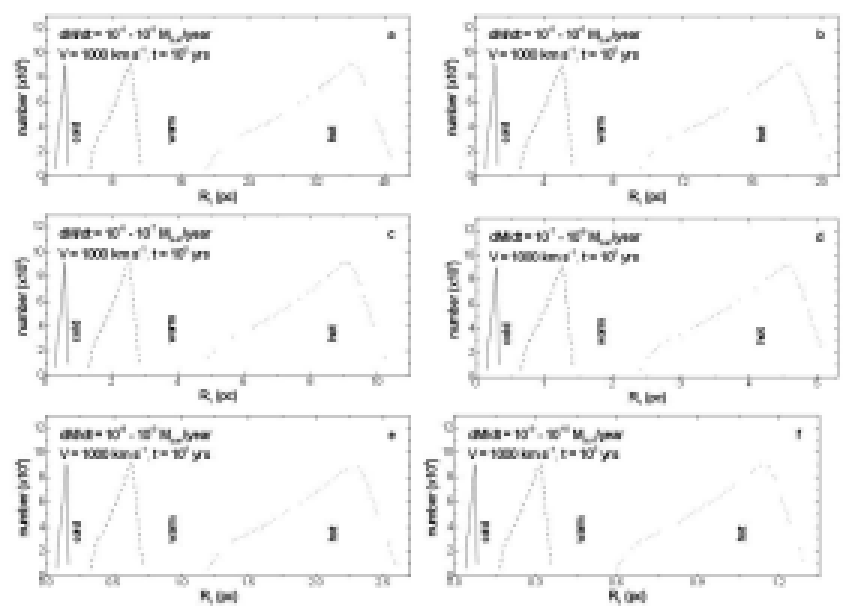

Figure 8: Size distribution of inner shocked region $\left(R_{1}\right)$ of the ISB formed due to PPN wind $\left(1,000 \mathrm{~km} \mathrm{~s}^{-1}\right)$ in cold (solid line), warm (dashed line) and hot (dotted line) ISM. The mass loss rates are given. 

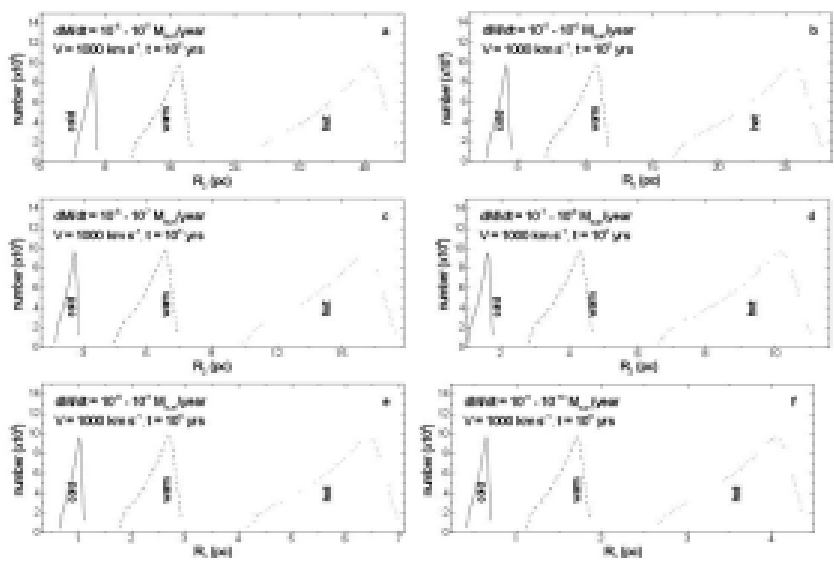

Figure 9: Size distribution of inner shocked region $\left(R_{2}\right)$ of the ISB formed due to PPN wind (1,000 $\left.\mathrm{km} \mathrm{s}^{-1}\right)$ in cold (solid line), warm (dashed line) and hot (dotted line) ISM. The mass loss rates are given.

The radius $R_{2}$ of the bubble for the mass loss rate $10^{-5}$ to $10^{-6}$ $\mathrm{M}_{\text {sun }}$ /year is 4.03 pc (Fig. 9b) for cold bubble. This turns 10.72 pc for warm and 25.41 pc for hot bubble. Similarly this radius for cold, warm, and hot medium for mass loss rate $10^{-6}$ to $10^{-7}$ $\mathrm{M}_{\text {sun }}$ /year are 2.54 pc, 6.76 pc, and 16.03 pc respectively (Fig. 9c). Thus the value of $R_{2}$ decreases as the mass loss rate of the PPN phase star decreases. A similar trend is observed for other mass loss rates. This suggests that the bubbles in the cold medium are stable than that in the hot medium.

The bubble radius $R_{2}$ due to the PPN phase stars (Fig. 9) is larger than that the AGB phase stars. This is due to the emission of high wind velocity ( 1,000 km/s) from the PPN phase star than that of the AGB phase stars ( $50 \mathrm{~km} / \mathrm{s})$. In Fig. 9, no overlapping of the distribution can be seen. The ratio $R_{2} / R_{1}$ is found to be $>1$ in all cases. In the case of cold ISM, this ratio goes up to 8 . The preferred mass loss rate from the PPN phase stellar wind lie in the range $10^{-4}$ to $10^{-6} \mathrm{M}_{\text {sun }}$ /year. Thus, the possibility of the existence of the interstellar bubble is high in the case of PPN phase. Here, the ratio $R_{2} / R_{1}$ is found to be $<$ 5.0 in all types of ISM. Thus, the bubble can be survived for the time scale $10^{5}$ years when the ambient ISM is triggered by the PPN phase stellar wind.

\section{RW Phase}

An example of relativistic wind phase is the pulsar nebula. A pulsar nebula is a synchrotron nebula powered by the relativistic wind of an energetic pulsar (Weiler \& Panagia 1978). At the early stage of their evolution, pulsar wind nebulae are often found inside the shells of supernova remnants. The prototype pulsar wind nebula is the Crab Nebula. Pulsar wind nebulae are believed to be powered by active rotation-powered pulsars, through a relativistic wind of particles and magnetic fields. The pulsar's wind is decelerated to a sub-relativistic speed across a strong standing shock (Chatterjee 2004). The emitted particles sweep out the interstellar gases and form bubbles. Figure 10 shows the sizes of the inner shocked region pumped by relativistic wind.
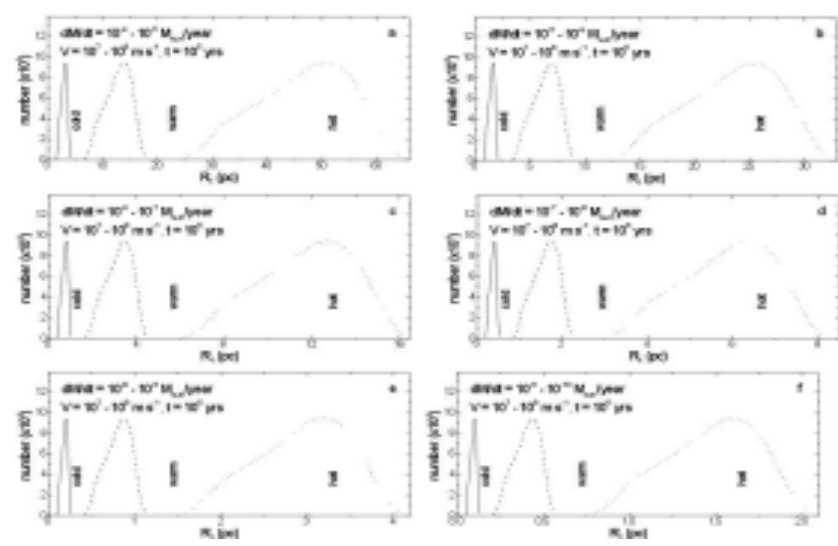

Figure 10: Size distribution of inner shocked region $\left(R_{1}\right)$ of the ISB formed due to relativistic wind $\left(\sim 10^{7}\right.$ to $\left.10^{8} \mathrm{~m} \mathrm{~s}^{-1}\right)$ in cold (solid line), warm (dashed line) and hot (dotted line) ISM. The mass loss rates are given.

The range of $R_{1}$ in the cold ISM becomes $1.55-4.08$ pc for the mass loss rate $10^{-4}$ to $10^{-5} \mathrm{M}_{\text {sun }}$ /year (Figs. 10a). The preferred value of $R_{1}$ is $\sim 3.15$ pc when the mass loss rate is extremely high ( $10^{-4}$ to $10^{-5} \mathrm{M}_{\text {sun }}$ /year) (Fig. 10a). This situation never becomes for the pulsar. The mass loss rate of the pulsar is extremely low. For the mass loss rate $10^{-6}$ to $10^{-7} \mathrm{M}_{\text {sun }}$ /year, the peak $R_{1}$ value becomes 0.79 pc, 3.44 pc and 12.55 pc for cold, warm, and hot mediums, respectively (Fig. 10c). For the mass loss rate $10^{-7}$ to $10^{-8} \mathrm{M}_{\text {sun }}$ /year, $R_{1}$ is found to be $0.41 \mathrm{pc}, 1.79$ pc, and 6.28 pc for cold, warm and hot mediums respectively (Fig. 10d). As the mass loss rate decreases, the $R_{1}$ value decreases. Thus, for $10^{-9}$ to $10^{-10} \mathrm{M}_{\text {sun }}$ /year, the size of the inner shocked region is smaller than 1 pc even for hot ISM. For the hot ISM, the FWHM is high. This suggests the less stability of the bubble for the evolution time $10^{5}$ year.
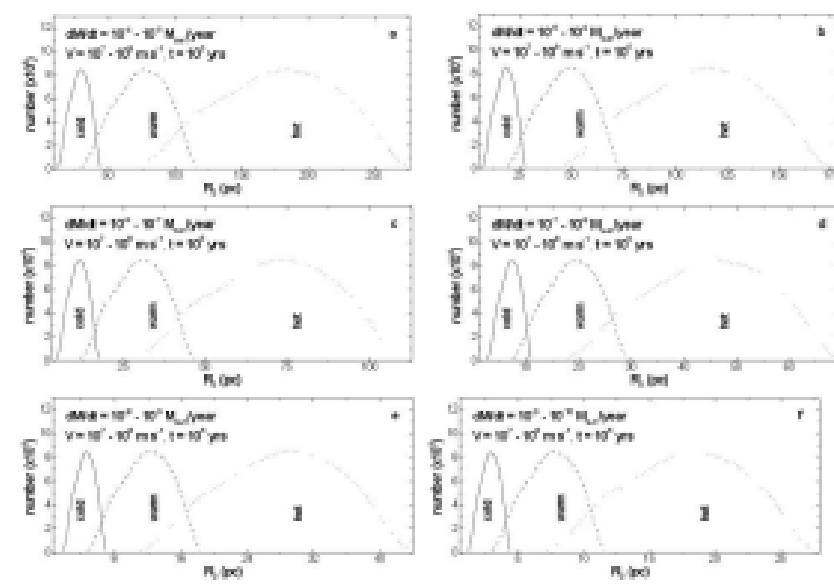

Figure 11: Size distribution of outer shocked region $\left(R_{2}\right)$ of the ISB formed due to relativistic wind ( $10^{7}$ to $10^{8} \mathrm{~m} \mathrm{~s}^{-1}$ ) in cold (solid line), warm (dashed line) and hot (dotted line) ISM. The mass loss rates are given.

Figure 11 shows the overall size of the ISB when it is formed due to the relativistic wind. For the mass loss rate $10^{-6}$ to $10^{-7}$ $\mathrm{M}_{\text {sun }}$ /year, the preferred R2 value becomes 11.24 pc, 29.93 pc, and 75.31 pc for cold, warm, and hot mediums, respectively (Fig. 10c). The distribution curves are found to overlap each 
other. The ratio $R_{2} / R_{1}$ is found to be $>5$. Thus the size of inner shocked region is small as compared to the size of the outer shocked region. Pulsar emits relativistic wind with low mass loss rate $\sim 10^{-10} \mathrm{M}_{\text {sun }}$ /year whereas carbon dwarf star emits relativistic wind with a high mass loss rate $\left(\sim 10^{-5} \mathrm{M}_{\text {sun }}\right.$ /year $)$. The carbon dwarf star behaves like a smoking gun. The flare star also emits relativistic wind with a moderate mass loss rate ( $\sim 10^{-7} \mathrm{M}_{\text {sun }}$ /year). Thus, the formation of ISB can not be denied in the RW phase. However, the stability of that structure is under question. We conclude that the bubble can be survived in the case of relativistic wind if the ISM is warm or hot.

\section{CONCLUSION}

We have discussed the results of the numerical simulations for the first $10^{5}$ years of the development of spherically symmetric interstellar bubbles (ISB). The model of the ISB as derived by Weaver et al. $(1975,1977)$ is used in our simulation. We have assumed three phase interstellar medium (ISM) model as proposed by Mckee \& Ostriker (1975) and estimated the size of the size of the inner shocked region and outer shocked region of the ISB. Our results are based on calculations including $10^{6}$ virtual stellar wind particles. We discuss the result in the context of Asymptotic Giant Branch (AGB) phase, Proto-Planetary Nebula (PPN) phase and Relativistic Wind (RW) phase.

\section{We summarize our results as follows:}

(1) The size of the interstellar bubble is found 0.14 pc to 9.03 pc in the case of AGB wind. This size increases for the PPN and RW phase up to $0.64-40.28$ pc and 2.83 pc - 178.12 pc, respectively.

(2) The ISB due to AGB wind can survive in the case of cold or warm interstellar medium. In the hot ISM, the ISB can not be formed due to the AGB wind. The bubble can be survived in the case of PPN phase stellar wind for all kinds of the ISM. We intend to work in this aspect in the future. In addition, we conclude that the bubble can be formed in the case of relativistic wind if the ISM is warm or hot. In the cold ISM, the ISB can not be formed due to the relativistic wind. These results are drawn from our numerical simulations.

(3) In the warm ISM, bubble can be formed due to the relativistic wind if the mass loss rate is extremely high. This is almost same with the case of the hot ISM. In few cases, our results contradict with each other. These results should be tested observationally in the future.

(4) In the warm ISM, the overall size of the bubble increases but do not exceed the recommended limit. Warm ISM is important in the sense that the bubble of all kinds can be survived. However, the hot ISM is not recommended for the good environment for the existence of ISB. The size of the bubble exceeds the recommended limit (i.e., 1 to $5 \mathrm{kpc}$ ) for both the PPN and pulsar phase. However, suitable AGB wind can trigger the environment for a bubble in the hot ISM.

The results of this work should be tested observationally. For this, study of HII region in the infrared sky provides the knowledge regarding the shocked interstellar region. Probably, this region represents the fossil of the interstellar bubble. A numerical time dependent hydrodynamic simulation would be the next step in order to understand new kind of interaction between the stellar wind and the ambient ISM.

\section{ACKNOWLEDGEMENTS}

This research has made use of the NASA/IPAC Extragalactic Database (NED) which is operated by the Jet Propulsion Laboratory, California Institute of Technology, under contract with the National Aeronautics and Space Administration. We acknowledge Prof. Walter Saurer, Prof. Stefan Kimeswinger, Prof. Udayraj Khanal and Dr. Jaturong Sukontochat for their valuable contributions and critical comments. One of the authors (AD) acknowledges Central Department of Physics, Tribhuvan University, Kirtipur, for providing various support for his masters (M.Sc.) thesis.

\section{REFERENCES}

[1] Weinberger R.\& Kerber F., Multidiciplinary Journal Science, 276,1382 (1997).

[2] Cox, D. P. 2005, Annual Review of Astronomy and Astrophysics, 43: 337.

[3] Weaver, R., McCray, J., Castor, J. 1977, Astrophysical Journal, 218: 377.

[4] McKee, C. F., Ostriker, J. P. 1977, Astrophysical Journal, 218: 148

[5] Castor, J., McCray, J., Weaver, R. 1975, Astrophysical Journal Lett., 200: L107.

[6] Odenwald, S. F. 1998, Astrophysical Journal, 325: 320.

[7] Dyson, J. E., de Vries J. 1972, Journnal Astronomy \& Astrophysics, 20: 223.

[8] Avedisova, V. S. 1972, Soviet Astr.- Astronomical Journal, 15: 708.

[9] Falle, S. A., Tucker, W. H. 1969, Astrophysical Journal, 157: 1157.

[10] Aryal B. \& Weinberger R. 2006, Journal Astronomy \& Astrophysics, 446: 213.

[11] Sahai, R., Sanchez, C. C., Morris, M. 2005, Astrophysical Journal, 620: 948.

[12] Weiler, K. W., Pangia, N. 1978, Journ. Astronomy \& Astrophysics, 70: 419.

[13] Chatterjee, S. \& Cordes, J. M. 2004, Astrophysical Journal, 600: L51.

\section{Appendix A: Programme file}

clear all; $\quad \%$ making the work place free of memory work place free of memory

$\mathrm{t}=$ cputime;

input('Type the name of your input file: ');

$27) * 10 \$ \wedge\{$ |wedge $\$\{6\}$;

$\mathrm{M}=\mathrm{m} * 1.99 * 10 \$ \wedge\{$ |wedge $\$(30) / 31536000 ;$

$\mathrm{V}=\mathrm{v}$;

$\mathrm{T}=\mathrm{t} * 31536000 ; \backslash 1$

$\mathrm{R} 1=(0.90 *(\mathrm{a} \$ \wedge\{$ \wedge $\$(3 / 2)) *((1 . / \mathrm{D}) . * \mathrm{M}) . \$ \wedge\{$ lwedge $\$(3 /$ $10) .{ }^{*} \mathrm{~V} . \$ \wedge\{\backslash$ wege $\}(1 / 10) * \mathrm{~T} \$ \wedge\{\backslash$ wedge $\left.\} \$(2 / 5)\right) /$ $\left(3.08^{*} 10 \$ \wedge\{\right.$ lwedge $\$(16))$; 
$\mathrm{R} 2=((0.406 / \mathrm{pi}) \$ \wedge\{$ |wedge $\$ \$(1 / 5) *((1 . / \mathrm{D}) . * \mathrm{M}) . \$ \wedge\{$ |wedge $\} \$(1 /$ $5) . * \mathrm{~V} . \$ \wedge\{\backslash$ wedge $\} \$(2 / 5) * \mathrm{~T} \$ \wedge\{\backslash$ wedge $\} \$(3 / 5)) /$ $(3.08 * 10 \$ \wedge\{$ lwedge $\$(16))$;

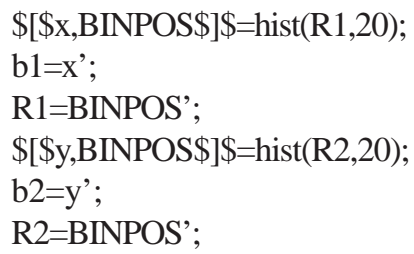

sprintf('Calculation time: f seconds ',cputime-t)

\section{Appendix B: An Example - Input files for AGB Stars in Cold Medium}

\% name of the input file: AGB1 \% Input file for cold ISM Isetlength $\{$ baselineskip $\}$ \{0.5 baselineskip $\}$

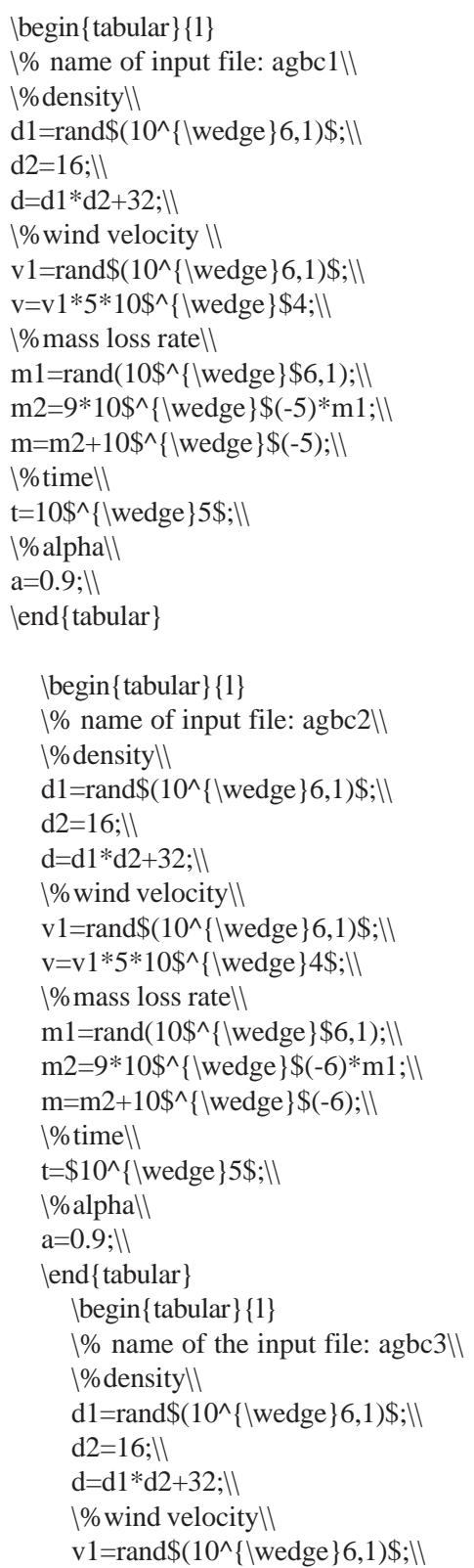

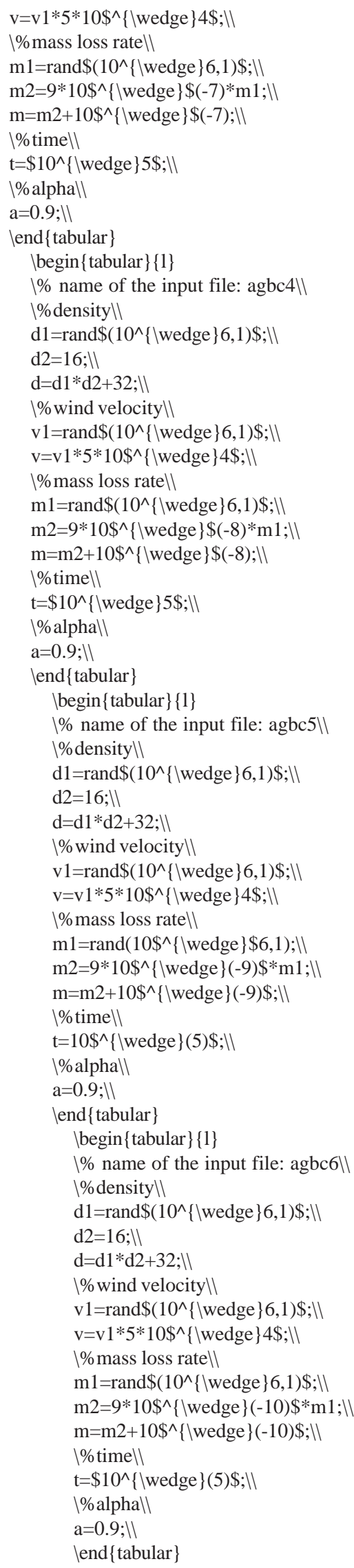

